Case report

\title{
Unilateral EEG sweat artifacts in a patient of brainstem hemorrhage with central Horner's syndrome
}

\author{
Pankaj Singh, A.R. Bansal* \\ Institute of Neurosciences, Medanta - The Medicity, Sector 38, Gurgaon, Delhi NCR, India
}

\section{A R T I C L E I N F O}

\section{Article history:}

Received 26 April 2016

Accepted 20 September 2016

Available online 31 October 2016

\section{Keywords:}

Sweat Artifact

Horner Syndrome

EEG artifact

\begin{abstract}
A B S T R A C T
We present an interesting EEG finding of persistent unilateral sweat artifacts in a 47 years old male patient with right hemiplegia secondary to left thalamic bleed with extension of hemorrhage into the left internal capsule, the left midbrain and ventricles. The EEG was done for altered sensorium. It showed unilateral (right sided) sweat artifacts throughout the EEG recording. The anhydrosis on left side of the face was due to left sided Horner's syndrome because of involvement of first order neurons in the left midbrain. This EEG finding is highlighted because of it being relatively less common but interesting finding.
\end{abstract}

(C) 2016 Published by Elsevier, a division of RELX India, Pvt. Ltd on behalf of Indian Epilepsy Society.

\section{Case study}

A 47 years old man, under regular treatment for hypertension presented to our institute with complaints of sudden onset drowsiness, right-sided weakness and slurring of speech. On examination, patient was drowsy but arousable with verbal commands. He had right-sided UMN facial weakness, right hemiparesis and hypoesthesia and right Horner's syndrome (right sided ptosis, miosis, enophthalmos and anhydrosis). He was detected to have acute intraparenchymal hemorrhage measuring $2.8 \times 2.18 \mathrm{~cm}$ in left thalamic region extending into posterior limb of internal capsule, left side of midbrain with intraventricular extension into the third ventricle, dependent occipital horn of bilateral lateral ventricles and fourth ventricle (Fig. 1a,b).

He was admitted to neurointensive care unit. Post stabilization patient was shifted to ward. While in ward, because of fluctuating sensorium patient was advised electroencephalogram (EEG) to rule out intermittent seizures or nonconvulsive status epilepticus.

His EEG showed exclusive right-sided sweat artifacts in the form of roughly $0.5 \mathrm{~Hz}$ medium amplitude waves (Fig. 2a). Another EEGs done next day had the similar findings (Fig. 2b). Patient had clinical features of left sided Horner's syndrome. The anhydrosis over left side of face lead to absence of sweat artifacts on left side.

\footnotetext{
* Corresponding author.

E-mail address: atmarambansal@yahoo.com (A.R. Bansal).
}

\section{Discussion}

Although EEG is aimed at recording the cerebral activity to look for any evidence for epileptiform discharges, it is not uncommon to find various artifacts during EEG recordings particularly if it is done at bed side. One of them is sweat artifacts which can be seen as low amplitude irregular asymmetrical extremely low frequency (usually $0.25-0.5 \mathrm{~Hz}$ ) waveforms oscillating across the baseline. Sweat leads to slow shift of electrical baseline by changing the impedance between the electrode and skin. ${ }^{1}$ It also loosens the contact between the electrode and skin. These artifacts are intermittent and usually bilateral but they can be found unilaterally also. Lowering the room temperature and wiping the area with ether or alcohol can reduce the sweating and prevent sweat artifacts.

Unilateral sweat artifacts persisting throughout the record is a less common finding and is peculiar to Horner's syndrome. In our patient, right sided sweat artifacts were seen throughout the recording. Absence of sweat artifacts on left side was secondary to anhydrosis caused by left Horner's syndrome. Other medical causes of unilateral sweating are underlying collection (e.g. subgaleal hematoma) in or under the skin, Harlequins syndrome, ${ }^{2}$ localized unilateral or segmental hyperhydrosis, ${ }^{3}$ Frey's auriculotemporal syndrome ${ }^{4}$ and Ross syndrome. ${ }^{5}$

\section{Conclusion}

We hereby report an interesting EEG finding with unilateral sweat artifacts in relation to Horner's Syndrome. 

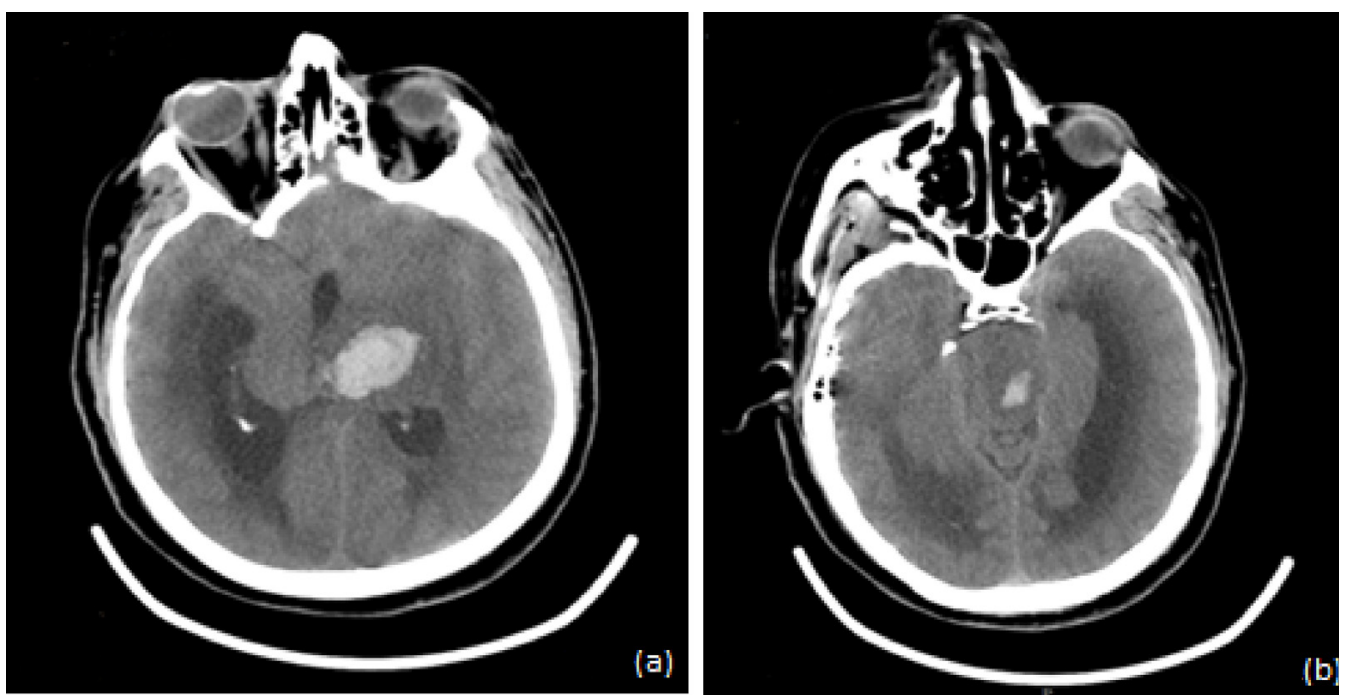

Fig. 1. The axial CT sections at the level of thalamus (a) and midbrain (b) showing the location of intraparenchymal hemorrhage on day 4 of stroke. It shows the extension of hypertensive bleed from the left thalamus into the left midbrain.
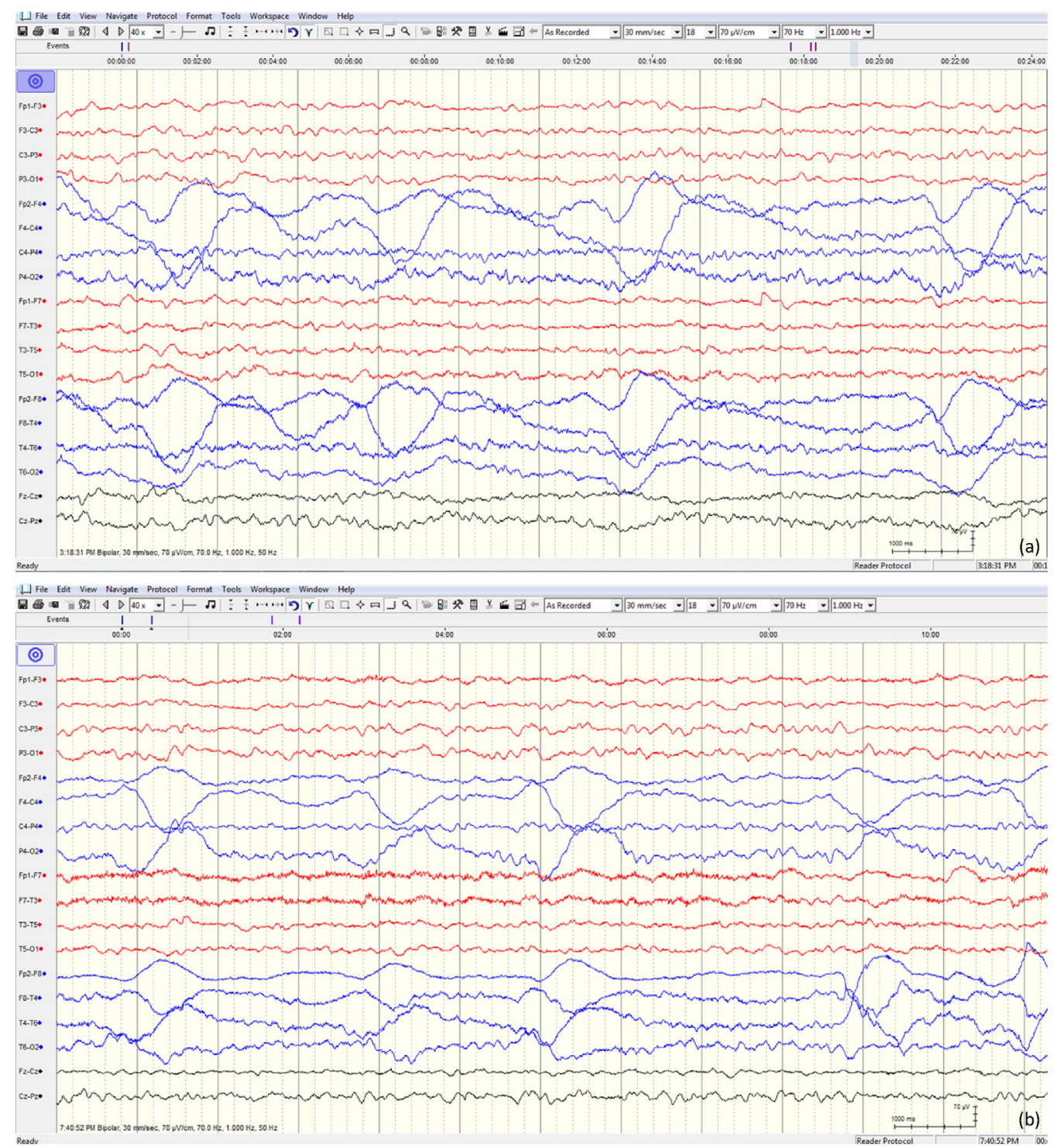

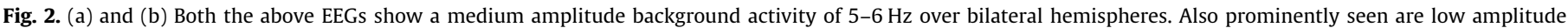

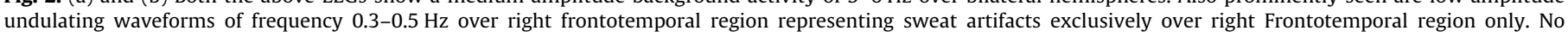
epileptiform discharges were seen. 


\section{Conflicts of interest}

The authors have none to declare.

\section{References}

1. Klass DW. The continuing challenge of artifacts in the EEG. Am J EEG Technol. 1995;35:239-269.
2. Lance JW, Drummond PD, Gandevia SC, Morris J. Harlequin syndrome: the sudden onset of unilateral flushing and sweating. I Neurol Neurosurg Psychiatry. 1988;51:635-642.

3. Yadalla HKY, Ambika H, Chawla S. A case of idiopathic unilateral circumscribed hyperhidrosis. Indian J Dermatol. 2013;58(2):163.

4. Prattico F, Perfetti P. Frey's Syndrome - images in clinical medicine. $N$ Engl J Med. 2006;355:66.

5. Yasar S, Aslan C, Serder ZA, Demirci GT, Tutkavul K, Babalik D. Ross syndrome: unilateral hyperhidrosis, Adie's tonic pupils and diffuse areflexia. J Dtsch Dermato Ges. 2010;8(12):1004-1006. 